\title{
The Drosophila p38 MAPK pathway is required during oogenesis for egg asymmetric development
}

\author{
Magali Suzanne, ${ }^{1}$ Kenji Irie, ${ }^{2}$ Bruno Glise, ${ }^{3}$ François Agnès, ${ }^{1}$ Eiji Mori, ${ }^{2}$ Kunihiro Matsumoto, ${ }^{2}$ \\ and Stéphane Noselli ${ }^{1,4,5}$

\begin{abstract}
${ }^{1}$ Centre de Biologie du Développement, UMR5547 Centre National de la Recherche Scientifique (CNRS), 31062 Toulouse Cedex, France; ${ }^{2}$ Department of Molecular Biology, Graduate School of Science, Nagoya University, and Core Research for Evolutional Science and Technology (CREST), Japan Science and Technology Corporation, Nagoya 464-0814, Japan; ${ }^{3}$ Developmental Genetics Programme, Sheffield, South Yorkshire S10 2TN, UK; ${ }^{4}$ Department of Genetics, Harvard Medical School, Boston, Massachusetts 02115 USA
\end{abstract}

In mammalian cells, the p38 mitogen-activated protein kinase (MAPK) pathway is activated in response to a variety of environmental stresses and inflammatory stimuli. However, the role of p38 MAPK signaling in unchallenged conditions remains largely unknown. We have isolated mutations in a Drosophila p38 MAPKK gene homolog, licorne (lic), and show that during oogenesis, lic is required in the germ line for correct asymmetric development of the egg. In lic mutant egg chambers, oskar mRNA posterior localization is not properly maintained, resulting in anteroposterior patterning defects in the embryo. Furthermore, lic loss-of-function in the germ line leads to reduced EGF receptor activity in dorsal follicle cells and ventralization of the egg shell. Both these defects are associated with a diminution of gurken protein levels in the oocyte. Our phenotypic data argue for a role of lic in a post-transcriptional regulation of the grk gene.

Furthermore, they show that in addition to the well-characterized Ras/Raf/ERK MAPK pathway acting in the follicle cells, another related signaling cascade, the p38 MAPK pathway, is required in the germ line for correct axes determination. These results provide the first genetic demonstration of an essential function for a p38 pathway during development.

[Key Words: Drosophila; p38; MAPK; signaling; oogenesis; Gurken]

Received January 22, 1999; revised version accepted April 20, 1999.

In metazoans, communication of cells with their immediate and remote environment is crucial for pattern formation, cell differentiation, and morphogenesis. All cell types have their survival and fate depending on cell signaling activities and most cells will experience activation by multiple signals that are transduced by distinct pathways. Among these, the highly conserved mitogenactivated protein kinase (MAPK) signaling cascade emerges as a widely used pathway that is induced following exposure to many different extracellular and developmental stimuli (Davis 1994; Herskowitz 1995; Kyriakis and Avruch 1996). Activation of MAPKs can lead to an awesome variety of cell responses including proliferation, differentiation, apoptosis, cell movement, stress and immune responses (Duffy and Perrimon 1996; Kyriakis and Avruch 1996; Symons 1996; Noselli 1998). The apparent pleiotropic role of MAPK pathways relies in part on the reiteration of a conserved module made of two generic kinases, a MAPKK and its MAPK target

${ }^{5}$ Corresponding author.

E-MAIL snoselli@rascal.med.harvard.edu; FAX (617) 432-7688.
(Cano and Mahadevan 1995; Kyriakis and Avruch 1996). Based on genetic, structural and biochemical studies, three main MAPK pathways can be distinguished, that is, the ERK, JNK, and p38 pathways (Davis 1994; Vojtek and Cooper 1995; Kyriakis and Avruch 1996).

Genetic studies in invertebrates have demonstrated clearly an essential function for the ERK (extracellular signal-related kinase) and JNK [Jun amino (스)-terminal kinase)] pathways during development (for review, see Dickson and Hafen 1994; Perrimon 1994; Noselli 1998). In mouse, the recent knockout of the MKK4 gene indicates an essential function for JNK signaling in vertebrate development, as well (Yang et al. 1997). However, although it has been shown that p38 signaling has a role in apoptosis and is activated by many environmental stress signals (Xia et al. 1995; Raingeaud et al. 1995, 1996; Han et al. 1997; Kawasaki et al. 1997; Kummer et al. 1997; Frasch et al. 1998), a function for p38 signaling in normal development has not yet been established.

To address this question, we performed interspecific complementation in yeast to isolate a Drosophila p38 MAPKK gene, which we named licorne (lic). Both in 
yeast and in cell cultures, lic can activate vertebrate p38 specifically, suggesting that at least some components of the p38 pathway are conserved in Drosophila. The isolation and phenotypic analysis of lic mutations indicate an essential and complex lic function during oogenesis. Here, we will focus on the role of lic during oogenesis for anterior-posterior (AP) and dorsal-ventral (DV) patterning of the egg.

In the fruitfly Drosophila, oogenesis provides a workable genetic model to study pattern formation and the establishment of asymmetry in the egg. Each egg develops in a highly organized egg chamber containing 16 germ cells organized in a cyst, of which 1 will differentiate into the oocyte. The germ-line cyst is surrounded by somatic follicle cells, which are organized in a monolayer epithelium. The development of the so-called egg chamber proceeds in several steps, including growth, changes in shape, cell migration, and patterning events (for a comprehensive review on oogenesis, see Spradling 1993). The polarity of the oocyte and the future embryo depends on several cell communication events between the germ line and the somatic tissues, which signal the specific localization of few determinants at the anterior [bicoid (bcd)], posterior [oskar (osk)], and dorsal [gurken (grk)] regions of the developing oocyte (for review, see Lehmann 1995; Ray and Schüpbach 1996).

Recent work has provided a unifying model in which two inductive events originating from the oocyte and directed toward the overlying follicle cells account for the sequential establishment of AP and DV axis during oogenesis (Gonzalez-Reyes et al. 1995; Roth et al. 1995; for review, see Ray and Schüpbach 1996). The oocyte signal is encoded by the TGF- $\alpha$ grk gene, whose products are tightly associated with the nucleus and move from an initial posterior to an anterodorsal position during oogenesis. On its way to the anterodorsal corner, grk will activate the epidermal growth factor receptor (EGFR) first at the posterior and then at the dorsal side of the dorsal follicle cells for their determination. One consequence of posterior follicle cell determination is the sending back of an as-yet unknown signal to the oocyte, leading to microtubule repolarization, nucleus anteriorward migration and asymmetric localization of the maternal determinants bcd, osk, and grk. When the oocyte nucleus reaches the anterodorsal corner of the oocyte, a second peak of grk activity determines dorsal follicle cells fate; thus, the DV axis of both the eggshell and the embryo (for review, see Ray and Schüpbach 1996; Anderson 1998). Despite this general view and the fact that several gene products have been identified that participate in these dynamic events, the biochemical interactions that take place are still poorly understood. In addition, genetic screens have revealed that several molecularly uncharacterized genes may have a role in these processes, leading to the notion that the development of the egg chamber is more complex and requires additional gene activities and regulatory mechanisms yet to be identified (Ghabrial et al. 1998).

We show here that lic mutations provoke polarity defects in the eggshell and embryo, as a result of reduced activity of two localized determinants, osk and grk. The phenotypic similarities between lic and grk mutations suggest that lic patterning defects arise from a reduction of grk activity at the posterior and dorsal poles of the oocyte, placing lic in a pathway required for post-transcriptional regulation of grk. These data show that the Drosophila p38 MAPK pathway is essential for oogenesis and provide a new link between the p38 MAPK pathway and asymmetric development that may be conserved in other metazoans.

\section{Results}

Isolation of lic

The yeast hog1 pathway mediates cellular responses to an increase in external osmolarity (Herskowitz 1995). Mammalian p38 has been shown to complement the high osmolarity-sensitive growth phenotype of the hog1 MAPK mutant. This complementation is dependent on the presence of pbs2, a MAPKK that functions as an upstream activator of hog1. These data suggest that the pbs2 MAPKK is required for the activation of mammalian p38 in yeast, raising the possibility that yeast could be used to identify the Drosophila MAPKK(s) activating p38.

We transformed a polymyxin B sensitivity (pbs) pbs $2 \Delta$ mutant (TM334; Fig. 1) expressing p38 with a Drosophila cDNA library and screened for growth on medium containing $1 \mathrm{M}$ sorbitol (high osmolarity). We obtained a total of 20 transformants capable of growth in the presence of sorbitol. Of these, six clones failed to restore highosmolarity resistance in the absence of p38 expression, indicating that they suppressed the $p b s 2 \Delta$ mutation in a p38-dependent manner. These six candidates defined two classes, as determined by restriction enzyme analysis, and we will focus here on one class representing clones of a unique gene, which we named lic (Fig. 1A).

\section{lic encodes a Drosophila p38 MAPKK homolog}

The complete sequence of a full-length lic cDNA shows an ORF encoding a putative 335-amino-acid protein (Fig. 1C). This novel Drosophila protein is related to the MAPKK family, with strongest sequence identity to human p38 activators MKK3 and MKK6 (Fig. 1D; Dérijard et al. 1995; Lin et al. 1995). The two known phosphorylation sites required for MAPKK activation are well conserved in LIC, leading to the conclusion that lic encodes a Drosophila MAPKK of the p38 MAPKK family. Recently, the Drosophila Genome Project and PCR-based approaches allowed the identification of DMKK3, a Drosophila homolog of human $M K K 3$ and $M K K 6$, and two related p38 protein kinases (Han et al. 1998a,b). On the basis of sequence identity and chromosomal location, we conclude that DMKK3 and lic correspond to the same gene.

Transformation of yeast with a kinase-negative form of LIC (LIC-KN; see Materials and Methods), resulted in the loss of complementation of the pbs $2 \Delta$ osmolarity phenotype, indicating that LIC kinase activity is re- 


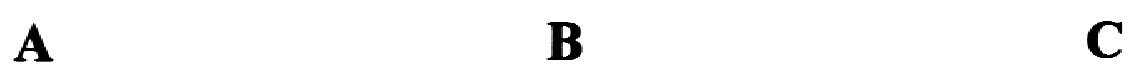

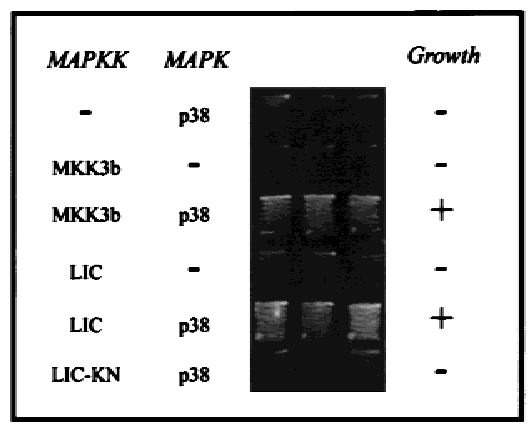
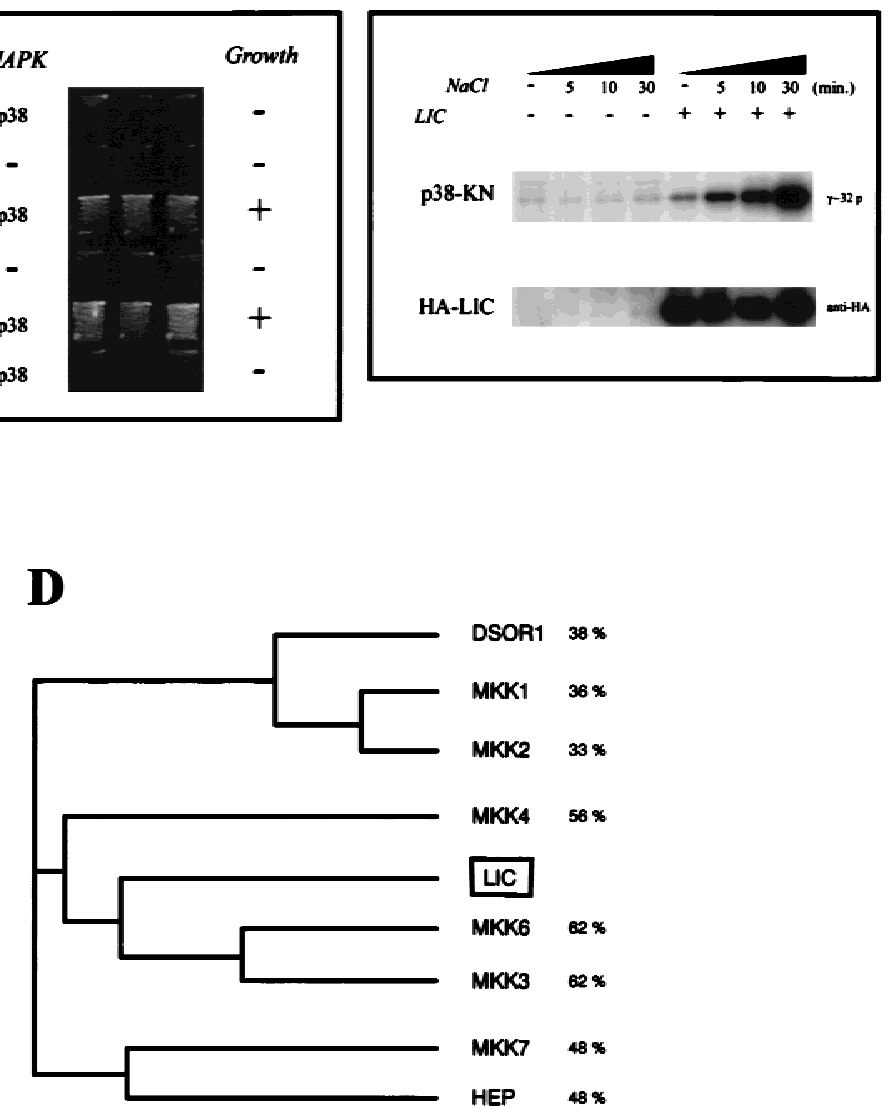

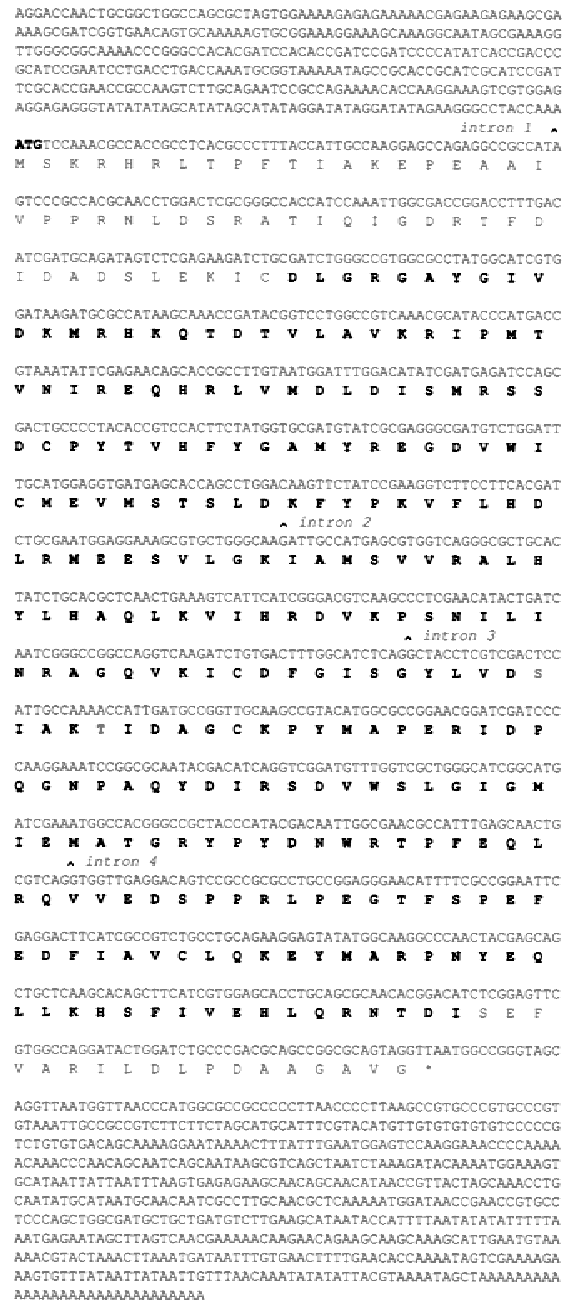

AAGAAAAAAAAAAAAAMARAB

Figure 1. Characterization of the lic gene. Complementation of a pbs2 deletion mutant in Saccharomyces cerevisiae using lic cDNA. (A) Yeast strain TM334 (pbs2) was transformed with various plasmids as indicated. Transformants were streaked onto YPD plates containing $1 \mathrm{M}$ sorbitol and incubated at $30^{\circ} \mathrm{C}$. (B) Yeast strain TM334 (pbs2) was transformed with empty vector $(-)$ or an expression vector for HA-LIC (+). Cells were treated with $\mathrm{NaCl}(0.5 \mathrm{M})$ for the indicated times. Immunoprecipitated complexes obtained with anti-HA were used for in vitro kinase reactions with GST-p38-KN as a substrate (top). The amounts of immunoprecipitated HA-LIC were determined with anti-HA (bottom). (C) Complete sequence of full-length lic cDNA (accession no. AJ238572) and a phylogenetic tree showing the relationship between lic and other Drosophila and human MAPKKs. lic is most homologous to the human MKK3 and MKK6 p38 activators $(D)$.

quired for suppression of the pbs $2 \Delta$ mutant phenotype (Fig. 1A).

To further examine LIC kinase activity, LIC was prepared as a glutathione $S$-transferase (GST)-LIC fusion protein in bacteria and purified. GST-LIC did not appreciably autophosphorylate or phosphorylate a catalytically inactive form of Xenopus p38 (GST-p38-KN; data not shown), suggesting that LIC is inactive when expressed in bacteria. We then tested the kinase activity of lic in yeast using influenza virus hemagglutinin-tagged LIC (HA-LIC). Expression of HA-LIC complemented a pbs $2 \Delta$ mutant expressing p38 (data not shown), indicating that this fusion protein is biologically functional. After exposure to osmotic shock, HA-LIC was immuno- precipitated from total cell extracts and lic activity was determined by an in vitro kinase assay using GST-p38$\mathrm{KN}$ as a substrate. As shown in Figure 1B, LIC phosphorylated p38-KN, whereas immunoprecipitates from yeast cells transformed with vector alone did not. Furthermore, osmotic shock stimulated the kinase activity of LIC in a time-dependent manner (Fig. 1B).

Taken together, these results demonstrate that LIC functions as a p38 activator, suggesting that LIC is involved in a p38 MAPK pathway in Drosophila.

\section{Isolation of lic mutations}

lic is located on the $\mathrm{X}$ chromosome, in close proximity to 
the hemipterous (hep) gene (11D1-2 on the cytological map). hep encodes a MAPKK of the JNKK family involved in epithelial morphogenesis during dorsal closure of the embryo (Glise et al. 1995; Glise and Noselli 1997). We took advantage of a $\mathrm{P}$ element inserted in the neighboring hep gene to generate small genomic deletions removing lic (see Materials and Methods). Screening of a total of 348 excisions allowed the recovery of 10 lethal double $\{$ hep, lic $\}$ mutants, as shown by complementation tests using a combination of ubiquitin (UB)-hep (UBhep) and UBlic transgenes (see Materials and Methods). Genomic Southern analysis confirmed the genetic data, revealing genomic deletions covering both genes (Fig. 2A; data not shown). We will focus here on two representative and independent alleles, H6 and G24.

Northern blot analysis and whole-mount in situ hybridization to embryos using a lic probe indicate a strong maternal contribution (Fig. 2B; data not shown). In ovaries, lic mRNAs are detected in the germ line from stage 7-8 onward (Fig. 2C). Expression is found in the nurse cells and mRNA accumulates in the oocyte by stage 1011 , although clear mRNA accumulation in the oocyte is only visible in later stages (12 and later). Interestingly, in the H6 and G24 double mutants, lic transcripts are not detectable, indicating that these mutations correspond to null or strong hypomorphic lic alleles (Fig. 2D). Expression of a lic cDNA under the control of the $u b$ promoter (UBlic construct) restores lic expression in mutant ovaries (Fig. 2E), thus allowing maternal rescue of the lic mutations (see below).

\section{lic affects $D V$ and $A P$ patterning}

The maternal expression of lic led us to study its function using germ-line clonal analysis of $\{$ hep, lic $\}$ double mutations (FLP-DFS technique; Chou and Perrimon 1992). To determine the phenotype(s) contributed by lic mutations, we performed a comparative analysis of \{hep, lic) germ-line clones induced in females expressing either UBhep, UBlic, or both UBhep and UBlic transgenes. Cuticle preparations of embryos laid by $\{h e p$, lic $\}$ or $\{h e p$, lic; UBhep\} germ-line clones (hereafter referred to as lic embryos) showed novel and complex phenotypes, as compared to those generated using a simple hep muta-
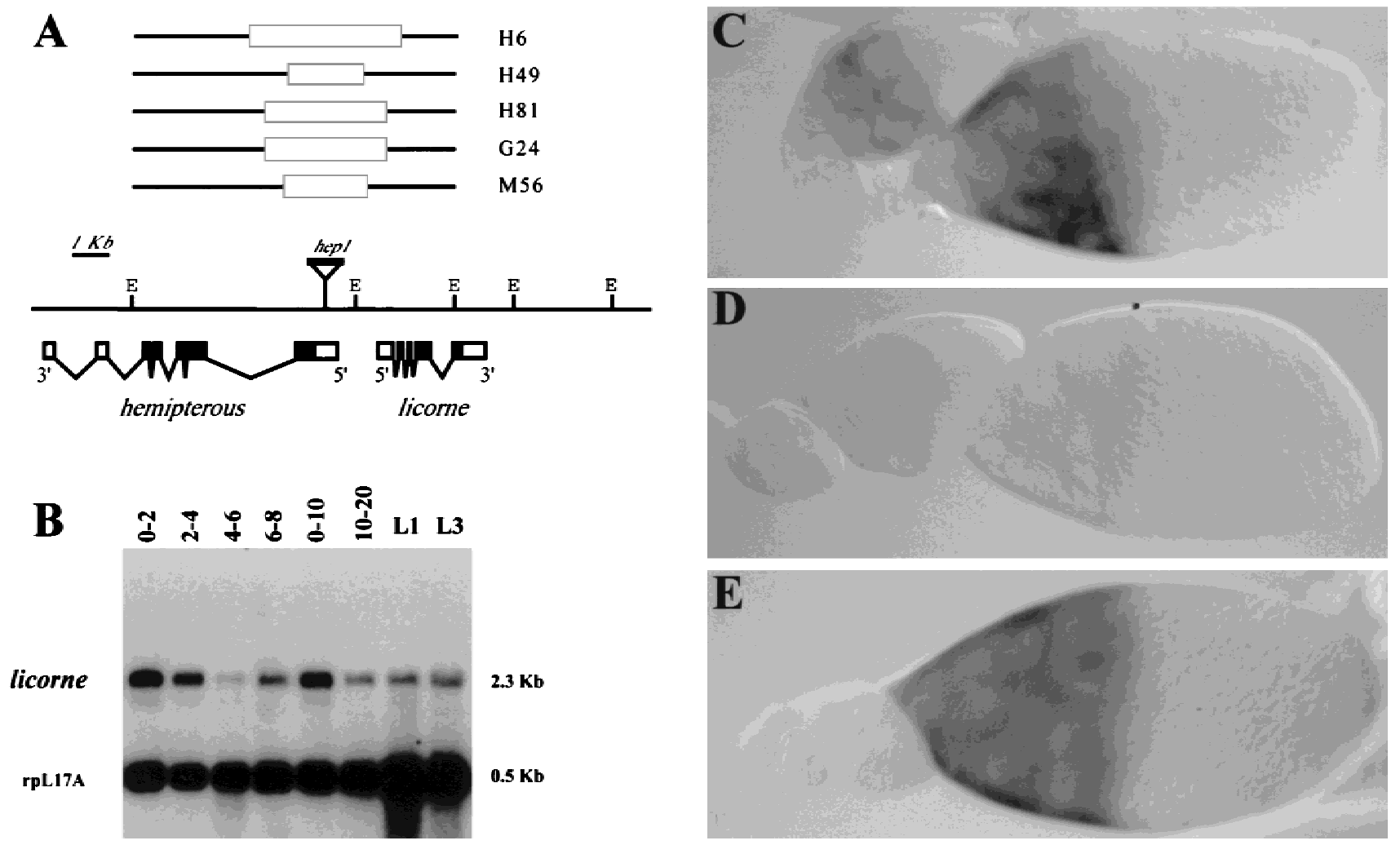

Figure 2. Molecular organization and expression of lic. (A) Genomic organization of the $\{$ hep, lic $\}$ locus. hep1 is a mobile P element inserted in the hep 5' untranslated region, and was used to generate double $\{$ hep, lic\} mutant alleles by imprecise excisions. The lines above the restriction map indicate the DNA lesions associated with five $\{$ hep, lic $\}$ alleles, as determined by Southern blot analysis. The solid boxes indicate DNA deletions within the two adjacent EcoRI fragments covering hep and lic. Below the restriction map is represented a schematic structure of full-length lic cDNA. Broken lines indicate the introns; solid boxes represent the coding region. (E) EcoRI. lic is expressed throughout development at variable levels. Expression in early (0-2 hr) embryos indicates a maternal contribution. The same blot was hybridized using a ribosomal gene probe (rpL17A) to assess mRNA levels. (C) In situ hybridization to wild-type ovaries indicates germ-line expression in nurse cells from stage 7-8 onward. $(D)$ In $\{$ hep, lic $\} \mathrm{H} 6$ germ-line clones, lic mRNAs are not detected. lic expression in the germ line can be restored using a UBlic transgene as shown in $E$. 
Figure 3. Chorion and cuticular phenotypes of lic. Dark-field photographs of chorion $(A, B)$ and embryo cuticles $(C-E)$ preparations of wild type $(A, C)$ and lic eggs or embryos derived from homozygous $\{$ hep, lic $\}$ germ-line clones $(B, D, E)$. The chorion in $B$ shows a ventralized phenotype as stated by fusion of dorsal appendages. (D) Partial deletion of the A4 abdominal segment indicates a weak abdominal segmentation defect. (E) In extreme cases, the whole abdominal region is deleted and eventually replaced by a single large denticle band. The terminal structures like the head skeleton and the Filzkörpers are normal. Anterior is on the left.
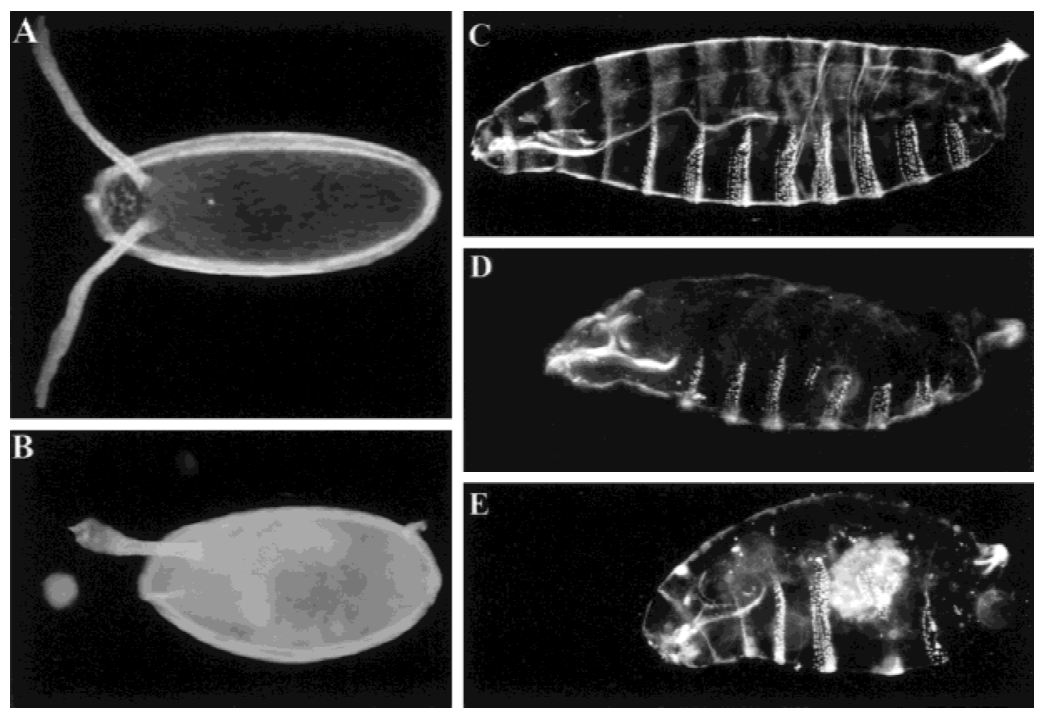

tion (Glise et al. 1995; data not shown). Most of the eggs $(80 \%-90 \%)$ appear not to be fertilized, are round in shape, and show a smaller size than the wild type, suggestive of incorrectly polarized eggs (Fig. 3B). In addition, the dorsal appendages, which mark the dorsal anterior region of the eggshell, are often shifted to a more dorsal position $(50 \%)$; in $\sim 20 \%$ of the eggs, dorsal appendages are fused, indicating a weak ventralization of the eggshell, reminiscent of some EGFR pathway hypomorphic mutants like grk and the EGFR (Fig. 3B). Finally, analysis of mutant egg chambers showed a highly penetrant 'dumpless' phenotype, that is, improper transfer of nurse cell content into the oocyte at the end of oogenesis (data not shown), a defect that may account for the reduced size of laid eggs (see Figs. 3 and 4).

Cuticle preparations of developed embryos (10\%-20\%) show variable but characteristic segmentation defects, consisting in the progressive deletion of abdominal segments (ranging from a partial deletion of segment A4 to a complete deletion of abdominal segments; Fig. 3D,E). This phenotype is reminiscent of that of the posteriorgroup genes, which control abdominal and pole cell development (see below). Because of the presence of a double mutation, some embryos show both a dorsal hole (due to the dorsal closure function of hep) and a deleted abdomen, indicative of an additive phenotype of the two MAPKK mutations (data not shown). When clones are induced in females expressing a UBhep transgene, the dorsal opening is completely rescued, confirming the hep origin of this phenotype. However, the unfertilized, ventralized, and segmental defects remain. In contrast, in embryos derived from $\{h e p$, lic; UBlic $\}$ clones, the proportion of unfertilized eggs decreases strongly (from $80 \%$ to $30 \%$ ), and eggshell ventralization defects or embryonic segmentation are no longer observed, even though the proportion of developing embryos is greatly increased.

These results indicate that the complex phenotypes associated with $\{$ hep, lic $\}$ chromosomes, including unfer- tilization and patterning defects, are a specific effect of lic loss of function.

\section{lic controls germ plasm assembly}

lic embryos show a segmentation phenotype that is reminiscent of the one produced by mutations in the maternal posterior-group genes, including osk, vasa, and nanos (for review, see St Johnston 1993; Rongo and Lehmann 1996). Most of the posterior-group genes can provoke both abdominal segmentation defects and a loss of germ cells, a dual defect that is due to the common localization of the posterior and germ cell determinants in the posterior germ plasm.

Like several posterior-group genes, lic embryos lack or have a strongly reduced number of pole cells, as shown using Vasa and Nanos as markers (Fig. 4B,E; data not shown). In lic mutants, Vasa protein fails to be accumu-

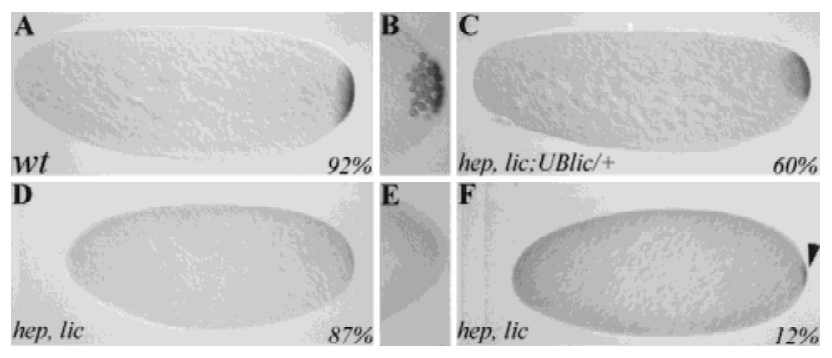

Figure 4. lic mutations affect pole plasm assembly. Immunostaining of wild-type $(A, B)$, \{hep, lic $\}(D-F)$, and $\{$ hep, lic; UBlic $\}$ $(C)$ embryos using an anti-vasa antibody. In wild type, the vasa protein is localized in a posterior crescent in early embryos $(A)$ and becomes incorporated into the pole cells once they form $(B)$. In lic mutant embryos, no $(D)$ or little $(F)$ vasa is present at the posterior pole, and no pole cells form $(E)$. These defects are due to a loss of lic function, as expression of a lic cDNA in the germ line using a UBlic transgene can restore vasa expression and posterior localization $(C)$. Anterior is on the left. 
lated at the posterior pole in most embryos $167 \%$; Fig. $4 \mathrm{D})$, although in some cases weak staining is observed (17\%; Fig. 4F). The defective posterior plasm and phenotypes lacking germ cells are observed in both $\{$ hep, lic\} and $\{$ hep, lic; UBhep $\}$ embryos. In contrast, expression of lic in the germ line $(\{h e p$, lic; UBlic $\})$ can restore normal Vasa staining and pole cell formation (Fig. 4C). We thus conclude from these experiments that most lic eggs (including a large proportion of unfertilized eggs) have a defect in germ plasm assembly.

lic has a role in abdominal segmentation, proper vasa protein and nanos mRNA localization at the posterior pole, and formation of the pole cells. These results suggest that lic also has a role in germ plasm assembly.

lic is required for the maintenance of osk $m R N A$ localization during oogenesis

The assembly of the germ plasm takes place during oogenesis and proceeds in several steps leading to the successive posterior localization of many different components (for review, see St Johnston 1993; Rongo and Lehmann 1996). A pivotal step in this process is the localization of the osk mRNA to the posterior pole of the oocyte in stage 8-9 egg chambers, which is the basis for the recruitment and assembly of downstream components like vasa and nanos. In lic germ-line clones, both osk mRNA expression and early posterior localization appear normal until stage 8 of oogenesis (data not shown). However, in stage 9 and older egg chambers, the osk mRNA is mislocalized, diffusing in the whole oocyte in a gradient from the posterior to the anterior pole (Fig. 5C). In later stages, osk transcripts are barely detectable, indicating that diffusion proceeds continuously in mutant egg chambers (Fig. 5D). A similar phenotype is observed in some osk missense mutants (known as the delocalizing alleles; Ephrussi et al. 1991; Kim-Ha et al. 1991), suggesting a role of Osk protein in the anchoring of its own mRNA at the posterior pole. Staining of lic mutant egg chambers using an anti-Osk antibody did not allowed detection of any reduction in Osk protein accu- mulation, thus indicating that lic affects osk mRNA localization independently of Osk translation (data not shown).

In some lic egg chambers, the mislocalized osk mRNAs also seem to partly accumulate in a more central position (Fig. 6D), reminiscent of the position of osk transcripts in mutants that have not reorganized the microtubules like EGFR pathway mutants. This result suggests that lic oocytes are not completely repolarized. However, we have not been able to detect any defect in the positioning of the nucleus, nor in the localization of a kinezin-lacZ microtubule-associated motor protein fusion (data not shown; Clark et al. 1994), suggesting that osk mislocalization is a more sensitive assay and lic defects are weak.

The correct localization of osk RNA at stage 8 and its later diffusion indicate that lic affects the maintenance of osk mRNA asymmetric localization in the oocyte (anchoring) rather than the mechanism of localization per se, most likely as a result of incomplete polarization along the AP axis.

\section{lic affects grk activity}

In addition to its requirement in AP patterning, lic mutations also affect the DV axis, as evidenced by ventralization of the eggshell (Fig. 3B). One important event in DV patterning is the correct localization of the grk ligand on the future dorsal side of the oocyte (see introductory paragraphs), a position that depends on the correct localization of the nucleus in the oocyte. Because we never observed mislocalized nuclei in lic mutant oocytes, lic DV defects are not likely to be the result of inappropriate nucleus migration or microtubule polarization. We thus asked whether the grk determinant itself might be affected in lic mutants. In situ hybridization using a grk probe did not detect any defect, suggesting that expression and localization of the grk mRNA are normal in lic mutant oocytes. However, immunostaining of egg chambers using an anti-Grk antibody showed reduction $15 \%$ at stage 10$)$ or mislocalization $(\sim 5 \%)$ of grk protein (Fig.

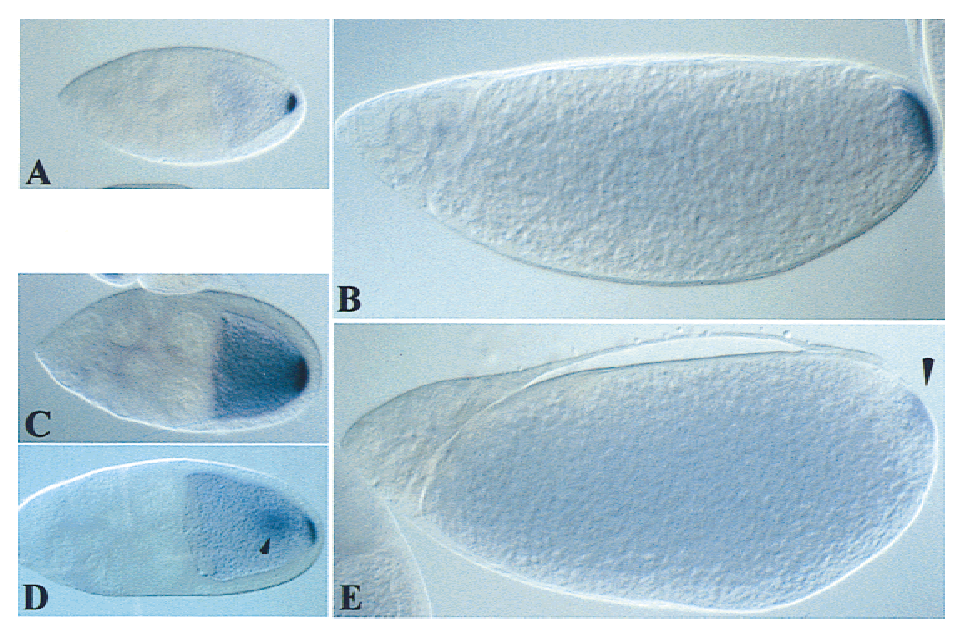

Figure 5. lic is required for the maintenance of osk mRNA posterior localization in the oocyte. In situ hybridization of wild-type $(A, B)$ and lic mutant $(C-E)$ oocytes at stages $9(A, C, D)$ and $12(B, D)$ of oogenesis. $(A, B)$ In wild type, osk mRNA is localized posteriorly in the oocyte until the end of oogenesis. $(C)$ In lic mutant egg chambers, osk mRNA normally localizes at the posterior pole of the oocyte but is not properly maintained and diffuse toward more anterior regions. (Arrowhead in $D$ ) In some cases, osk mRNA also accumulates centrally. (Arrowhead in E) osk transcripts are barely detectable in stage 12 oocytes, indicating that diffusion of osk proceeds continuously throughout oogenesis. Anterior is on the left. 
Figure 6. lic controls grk activity in mid-oogenesis. Immunostaining of wild-type $(A)$ and lic $(D, G)$ stage 9 oocytes using an anti-grk antibody (lateral views; dorsal is up). (D) In mutant oocytes, grk protein is mislocalized. The egg chamber in $G$ represents the most extreme localization defect that we have observed. $\beta$-Galactosidase staining of wild type $(B)$ and lic $(E, H)$ stage 10 egg chambers shows kek expression in the dorsal region of the follicle cells $(45 \% ; n=49)$. Note that in lic mutants, kek expression is affected, both in terms of levels and shape of the expression domain. In rare cases, kek expression is expanded laterally and ventrally, suggesting a dorsalization of the future egg chorion $(I ;<5 \%) .(C, F, I)$ The chorion phenotypes corresponding to grk and kek expression patterns in wild-type $(C)$ and lic egg chambers $(F, I ; 50 \%$ and $<1 \%$, respectively; $n=219 \mid . B, C, E, F$, and $I$ are dorsal views. $H$ is a lateral view, with dorsal up. For all panels, anterior is on the left.

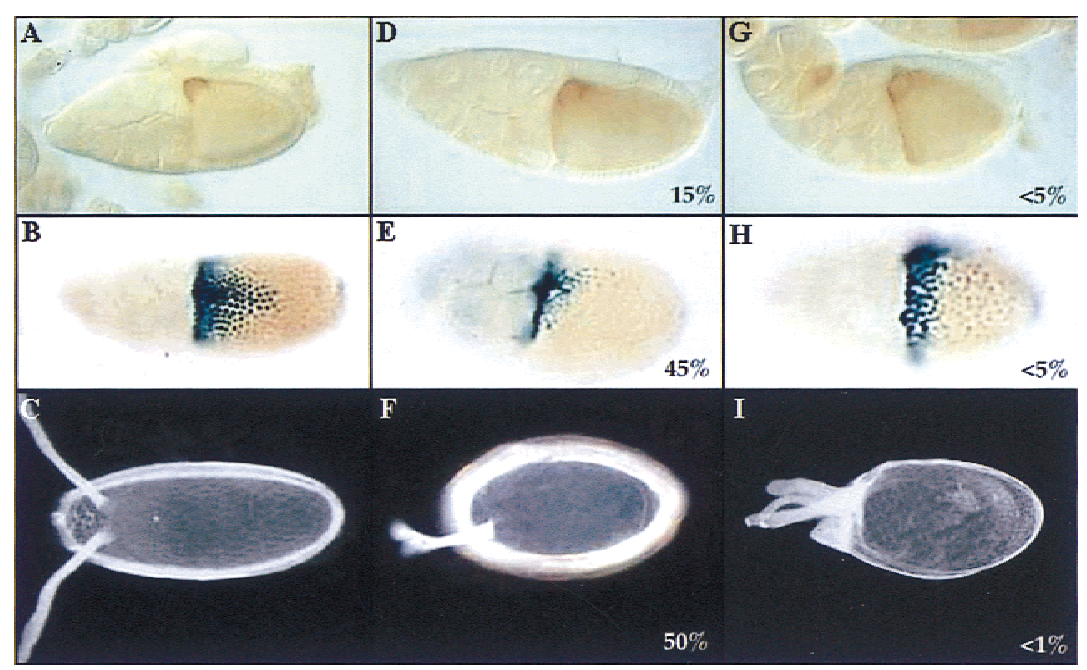

6D,G). To further characterize a loss of grk activity in lic oocytes, wild-type and mutant ovaries were stained using a kekon (kek)-lacZ reporter construct. The kek gene is a target of the EGFR in the follicle cells and thus serves as an indirect and sensitive assay to measure grk activity in the oocyte. In wild-type egg chambers, kek is expressed in dorsal follicle cells in a characteristic graded pattern reflecting both the intensity and localization of the underlying grk signal (Fig. 6C; Musacchio and Perrimon 1996; Sapir et al. 1998). In 50\% stage 10 lic mutant egg chambers, kek expression is reduced dramatically, as shown by a reduction in the number of responding follicle cells and a change in the shape of the kek expression domain (Fig. 6E). In rare cases $(<5 \%)$, we also observed an expansion of the kek signal in more lateral and ventral positions, an observation that might suggest a partial delocalization of grk activity in the oocyte. Consistent with this result, dorsalization of the chorion was observed in very rare cases $(<1 \%$; Fig. 6I).

Thus, lic loss of function in the germ line reduces EGFR activity in the dorsal follicle cells, most likely as a result of a reduction of grk activity in the oocyte.

\section{Discussion}

We report here the isolation and genetic analysis of lic, a Drosophila member of the family of p38 MAPKKs. Our data show that a novel, germ-line-specific MAPK pathway is required during oogenesis in several processes, including having an important role in the patterning of the egg. This is the first genetic demonstration of an essential function for a p38 MAPK pathway in development.

\section{p38 MAPK pathway in Drosophila}

The discovery of several related MAPK pathways in eukaryotes raises the important question of their respec- tive role during development. To address this question genetically, we have identified members of the MAPKK family in Drosophila (Glise et al. 1995; this study). Here, we show that a novel MAPKK gene, lic/DMKK3, encodes p38 MAPKK most homologous to the human $M K K 3$ and MKK6 genes. Using heterologous complementation in yeast, we show that lic is a p38 MAPKK capable of activating vertebrate p38 substrates. In addition, other experiments conducted in vitro and in cell cultures have shown that DMKK3/LIC can phosphorylate the two Drosophila Dp38a and Dp38b proteins. Altogether, these experiments show that a p38 pathway exists in Drosophila (Han et al. 1998a,b). In addition, these data indicate that, as in mammals, at least three different MAPK pathways exist in Drosophila (ERK, JNK, and p38), making this organism unique for studying MAPK signaling by genetic means.

The lic/Dp38K and hep/DINKK genes are clustered in the same locus, suggesting that these related genes may have a common ancestral origin. Studies in mammalian cells have shown that p38 and JNK pathways can be activated by identical stress stimuli (for review, see Kyriakis and Avruch 1996), indicating that these related pathways might also work together in some developmental processes. However, our functional analysis of hep and $\{h e p$, lic\} mutants show that each JNKK and p38 MAPKK gene has derived specific functions, leading to specific loss-of-function phenotypes. hep controls morphogenesis of the lateral ectoderm during dorsal closure of the embryo, without affecting AP patterning (Glise et al. 1995; Glise and Noselli 1997). As shown here, lic loss of function mutations affect asymmetric development during oogenesis, with phenotypic consequences on DV and AP patterning of the eggshell and the embryo. Because lic and hep are involved in different processes and the double mutant leads to additive phenotypes, it thus appears that in Drosophila the JNK and p38 MAPK pathways act independently during embryonic development. 
However, it is still possible that, as in vertebrates, stressful stimuli may be able to activate both Drosophila p38 and JNK pathways, an issue that can now be addressed genetically using lic and hep mutations.

The requirement for lic function in the oocyte raises the question of how p38 is activated. lic phenotypes are observed in a peculiar cell, the oocyte, whose nucleus is mostly transcriptionally silent (Spradling 1993). So far, all MAPK pathways have been shown to modulate gene activity in response to several stimuli. If lic does regulate transcription in the germ line, then a likely possibility is that these nuclear events take place in the nurse cells and that newly expressed gene products are transmitted to the oocyte through the ring canals. This may represent a unique and novel mechanism of MAPK signaling.

One puzzling observation is that the polarity defects associated with lic mutations are not fully penetrant (80\% of embryos show vasa localization defects; $50 \%$ of egg chorions show a ventralization phenotype), despite the fact that the lic mutants described here are likely to be null alleles. Although variable phenotypes are commonly observed among oogenesis genes, their origin is not well understood. In the case of lic, which belongs to the stress-activated family of MAPK pathways (Xia et al. 1995; Raingeaud et al. 1995, 1996; Kyriakis and Avruch 1996; Kawasaki et al. 1997; Kummer et al. 1997), it is possible that its loss of function will become more dramatic in stressful conditions, a view that is in part supported by the fact that lic phenotypes are cold sensitive (data not shown). Another, nonexclusive possibility is that partial redundancy may occur. This is supported by the fact that two different Drosophila p38-encoding genes have been identified, Dp38a and Dp38b (Han et al. $1998 \mathrm{a}, \mathrm{b})$, a situation that is unique in flies, as only one MAPK was identified that functions in the other ERK and JNK pathways. Given that only one p38 activator gene has been cloned so far (Iic/DMKK3), we cannot exclude the existence of another related function, and/or the possibility that lic may only activate one Dp38 MAPK in vivo. A way to address these issues will be to compare $D p 38 a$ and $D p 38 b$ single and double mutant phenotypes, once these will be available.

\section{p38 and ERK MAPK pathways interact during oogenesis}

A series of elegant genetic studies have demonstrated a clear link between AP and DV axis establishment and the activation of the EGFR in specific follicle cells. These patterning activities rely on cell communication between two different cell populations in the egg chamber, the germ-line cyst and the surrounding somatic follicle cells (Gonzalez-Reyes et al. 1995; Roth et al. 1995; for review, see Ray and Schüpbach 1996). EGFR activation in the follicle cells is triggered by the activity of grk in the underlying oocyte, and farther downstream signal transduction is mediated by the well-characterized ras/ raf/ERK MAPK pathway (Wasserman and Freeman 1998; for review, see by Ray and Schüpbach 1996). Here, we show that the p38 MAPK pathway also participates in asymmetric development of the egg chamber. Interestingly, ERK and p38 signaling are not active in the same cells, as lic function is restricted to the germ line, whereas ERK is only active in the follicle cells. The loss of function of lic or ERK lead to similar DV phenotypes, suggesting an interaction between these two MAPK pathways (see below). This view is well supported by the observation that grk activity and localization are affected in lic mutants (Fig. 6). Altogether, our results indicate that patterning of the egg relies on the activation of both the p38 and ERK pathways, representing the first example of cell communication based on activation of two different MAPK pathways in two distinct and apposing cell types (Fig. 7).

The observation that both axes are affected in lic mutants and that the phenotypes resemble in part those of weak grk mutants is very suggestive of a common origin for these defects, that is, a reduced grk activity not only on the dorsal side, as reported here, but also at the posterior pole. osk mislocalization in lic mutants is reminiscent of what can be observed in a weak allele of the gene encoding the grk receptor (EGFR; Gonzalez-Reyes et al. 1995; Roth et al. 1995; T. Schüpbach, pers. comm.). Although grk mRNA localization appears normal in lic egg chambers, we observed changes in grk protein localization and levels that correlate well with reduced kek activity in the dorsal follicle cells (Fig. 6). These results indicate that lic is required for correct GRK post-transcriptional regulation, at least on the dorsal side of the oocyte. We propose that lic also affects grk activity at the posterior pole of the oocyte, with consequences on the maintenance of osk mRNA localization (Fig. 5). Interestingly, several genes have been shown to be involved in the control of grk mRNA or protein localization and grk translation [aubergine (aub); cornichon (cni); encore; K10; maelstom; okra; orb; spinB; spinD; vasa; Roth et al. 1995; Neuman-Silberberg and Schüpbach 1996; Wilson et al. 1996; Clegg et al. 1997; Hawkins et al. 1997; Ghabrial et al. 1998; Styhler et al. 1998; Tomancak et al. 1998), thus defining a pathway that is important for post-

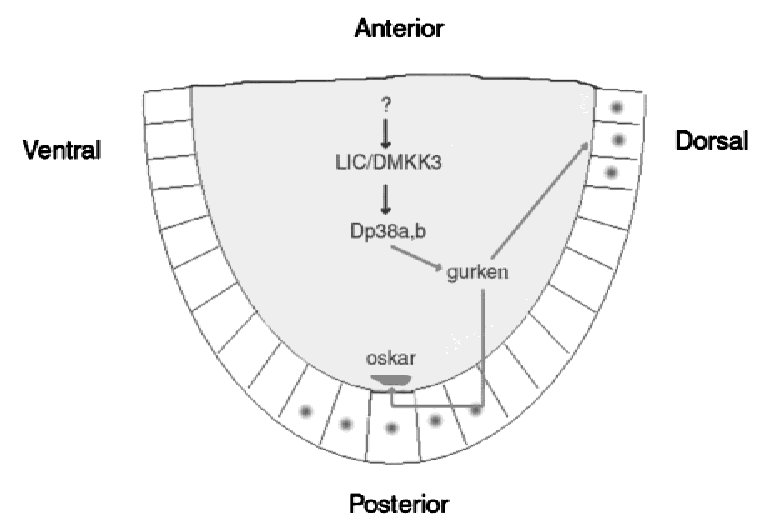

Figure 7. Model of p38 MAPK pathway function during oogenesis. The germ line (nurse cells and the oocyte), the site of lic/ p38 activity, is shaded; dots show the sites of EGFR activity in the follicle cells (posterior and dorsal). (See Discussion for details.) 
transcriptional regulation of grk and subsequent EGFR signaling. In these mutants, aspects of grk function are altered, leading to AP and DV defects reminiscent of those observed in lic. However, in contrast to grk, EGFR, and the upstream grk regulators mentioned above, lic mutations appear not to affect DV patterning of the embryos as assayed using the mesodermal marker twist (data not shown). This might argue for a more specific role of lic in DV patterning. Alternatively, it might reflect the partial redundancy discussed above and the weaker effect of lic on grk activity and patterning. For example, posterior osk mislocalization defects can be classified as weak as compared to strong grk, EGFR, cni, encore alleles: in lic, osk mRNA showed diffusion and central localization (Fig. 5), but osk transcripts are localized correctly at the posterior pole at stage 9, indicative of a weak polarization phenotype. Also, bcd and a Kinezin-LacZ fusion protein were localized correctly in lic, whereas in strong EGFR pathway mutants, bcd mRNA can be found at the posterior pole and the Kinezin-LacZ fusion at the center of the oocyte, like osk. Altogether our data thus argue for a role of lic in a post-transcriptional pathway regulating grk activity in the entire oocyte. However, and although lic does not affect osk translation (data not shown), we cannot rule out the possibility that lic may also affect anchoring of the osk mRNA at the posterior pole independently of the grk signal.

In vertebrates, few members of the p38 MAPK pathway have been identified, including the transcription factors ATF-2 (Dérijard et al. 1995; Rainjeaud et al. 1995) and MEF2 (Han et al. 1997), the MAPK/AP2 protein kinases and the Hsp25/Hsp27 proteins (Freshney et al. 1994; Rouse et al. 1994). The finding that p38 phosphorylates the small heat shock protein Hsp27 in rat ovarian granulosa cells for their rounding/aggregation (Maizels et al. 1998) provides a link between granulosa cells and Drosophila oogenesis development that will be interesting to investigate. Interestingly, Drosophila Hsp27 is expressed in nurse cells and the oocyte in a dynamic pattern, suggesting that regulatory events occur to modulate Hsp27 localization in the absence of heat shock (Marin and Tanguay 1996).

In conclusion, understanding how grk is activated together with the isolation of additional new members of the p38 pathway will provide a way to address specific models and gain access to the mechanisms whereby the p38 MAPK cascade regulates localized determinants during oogenesis.

\section{Materials and methods}

\section{Genetics}

A description of genetic markers and chromosome balancers can be found in Lindsley and Zimm (1992). The P-element hep1 insertion was mobilized using a stable source of $\mathrm{P}$ transposase, as described previously (Glise et al. 1995). A total of 348 independent excision events were selected and tested for X-linked lethality. Seventy-five lethal lines were recovered, and genetic rescue experiments were performed using UBhep, UBlic, or a combination of UBhep and UBlic transgenes. This approach allowed the identification of three different lethal groups: 63 corresponded to novel hep mutations, as expected of a starting P element located within the 5' UTR of hep; 10 corresponded to double $\{$ hep, lic $\}$ mutations, as indicated by their unique requirement of both UBhep and UBlic transgenes to be rescued; 2 corresponded to lethal mutations that are not rescued by any combination of UBhep and UBlic transgenes, and thus represent deletions that may remove additional gene(s).

Homozygous germ-line clones for $\{$ hep, lic $\}$ were induced using the FLP-DFS technique (Chou and Perrimon 1992), as described previously (Glise et al. 1995). For maternal rescue of $\{h e p$, lic $\}$ double mutant germ-line clones were induced in females carrying either UBhep ly w hep lic FRT101/y w ovoD1 FRT101; UBhep/MKRS, HS-flp), UBlic (y w hep lic FRT101/y $w$ ovoD1 FRT101; UBlic/MKRS, HS-flp) or a combination of UBhep and UBlic transgenes (y w hep lic FRT101/y w ovoD1 FRT101; UBhep, UBlic/MKRS, HS-flp).

\section{Immunohistochemistry and in situ hybridization}

Staining of embryos and egg chambers were performed as in Lasko et al. (1990) and Glise and Noselli (1997).

\section{Molecular biology and yeast expression}

Screening of Drosophila genes in yeast Yeast strains TM334 (MATa pbs2::HIS3 ura3 leu2 trp1 his3) expressing mammalian p38 was transformed with a Drosophila cDNA library constructed in the yeast expression vector $\mathrm{pDB} 20$, in which expression of the cDNA is under the control of the $A D H 1$ promoter. Osmoresistant transformants were selected by growing on YPD plates containing $1 \mathrm{M}$ sorbitol.

Construction of HA-lic The yeast expression vector for HA epitope-tagged lic was constructed by inserting a 1.0-kb BamHIEcoRV fragment containing the full-length lic cDNA into the BamHI-SmaI sites of pRS316-GAL-HA. The HA epitope recognized by the monoclonal antibody $12 \mathrm{CA} 5$ was attached inframe to the DNA encoding the amino terminus of lic. The HA-lic construct was expressed from the GAL1 promoter.

Assays for kinase activity in yeast cells Yeast cell extracts were prepared with a lysis buffer solution as described in Irie et al. (1994) and centrifuged for $30 \mathrm{~min}$ at 100,000g. The supernatant was subjected to immunoprecipitation with antibody to $\mathrm{HA}$ and the immune complexes were assayed for kinase activity. The activities of immunoprecipitated lic were assayed by its ability to phosphorylate recombinant GST-tagged catalytically inactive (KN) p38 (GST-p38-KN).

The UBhep construct has been already described (Glise et al. 1995). The UBlic construct was made by introducing a $4.5-\mathrm{kb}$ NotI fragment containing UBlic cDNA-Hsp70 3' UTR into the NotI site of the pCaSpeR4 transformation vector. Several independent UBlic transgenic lines were tested and showed rescue of lic zygotic lethality.

\section{Acknowledgments}

We thank A. Ephrussi, N. Perrimon, and T. Schüpbach for Drosophila stocks. We are grateful to P. Lasko, T. Schüpbach, and Y.N. Jan for providing various materials and reagents, and $\mathrm{T}$. Schüpbach for discussions. We thank L. Dubois, D. Cabannes, R. Catoira, H. Barcelo, and Giichi Takaesu for their initial help 
in this project. This work is supported by CNRS, Association pour le Recherche sur le Cancer, and Ligue Contre le Cancer.

The publication costs of this article were defrayed in part by payment of page charges. This article must therefore be hereby marked 'advertisement' in accordance with 18 USC section 1734 solely to indicate this fact.

\section{References}

Anderson, K.V. 1998. Pinning down positional information: Dorsal-ventral polarity in the Drosophila embryo. Cell 95: 439-442.

Cano, E. and L.C. Mahadevan. 1995. Parallel signal processing among mammalian MAPKs. Trends Biochem. Sci. 20: 117122.

Chou, T.B. and N. Perrimon. 1992. Use of a yeast site-specific recombinase to produce female germline chimeras in Drosophila. Genetics 131: 643-653.

Christerson, L.B. and D.M. McKearin. 1994. orb is required for anteroposterior and dorsoventral patterning during Drosophila oogenesis. Genes \& Dev. 8: 614-628.

Clark, I., E. Giniger, H. Ruohola-Baker, L.Y. Jan, and Y.N. Jan. 1994. Transient posterior localization of a kinesin fusion protein reflects anteroposterior polarity of the Drosophila oocyte. Curr. Biol. 4: 289-300.

Clegg, N.J., D.M. Frost, M.K. Larkin, L. Subrahmanyan, Z. Bryant, and H. Ruohola-Baker. 1997. maelstrom is required for an early step in the establishment of Drosophila oocyte polarity: Posterior localization of grk mRNA. Development 124: 4661-4671.

Davis, R.J. 1994. MAPKs: New JNK expands the group. Trends Biochem. Sci. 19: 470-473.

Dérijard, B., J. Raingeaud, T. Barrett, I.H. Wu, J. Han, R.J. Ulevitch, and R.J. Davis. 1995. Independent human MAP kinase signal transduction pathways defined by MEK and MKK isoforms. Science 267: 682-685.

Dickson, B. and E. Hafen. 1994. Genetics of signal transduction in invertebrates. Curr. Opin. Genet. Dev. 4: 64-70.

Duffy, J.B. and N. Perrimon. 1996. Recent advances in understanding signal transduction pathways in worms and flies. Curr. Opin. Cell Biol. 8: 231-238.

Frasch, S.C., J.A. Nick, V.A. Fadok, D.L. Bratton, G.S. Worthen, and P.M. Henson. 1998. p38 mitogen-activated protein kinase-dependent and -independent intracellular signal transduction pathways leading to apoptosis in human neutrophils. J. Biol. Chem. 273: 8389-8397.

Ephrussi, A., L.K. Dickinson, and R. Lehmann. 1991. oskar organizes the germ plasm and directs localization of the posterior determinant nanos. Cell 66: 37-50.

Freshney, N.W., L. Rawlinson, F. Guesdon, E. Jones, S. Cowley, J. Hsuan, and J. Saklatvala. 1994. Interleukin-1 activates a novel protein kinase cascade that results in the phosphorylation of Hsp27. Cell 78: 1039-1049.

Ghabrial, A., R.P. Ray, and T. Schüpbach. 1998. okra and spindle-B encode components of the RAD52 DNA repair pathway and affect meiosis and patterning in Drosophila oogenesis. Genes \& Dev. 12: 2711-2723.

Glise, B., H. Bourbon, and S. Noselli. 1995. hemipterous encodes a novel Drosophila Map kinase kinase, required for epithelial cell sheet movement. Cell 83: 451-461.

Glise, B. and S. Noselli. 1997. Coupling of Jun amino-terminal kinase and Decapentaplegic signaling pathways in Drosophila morphogenesis. Genes \& Dev. 11: 1738-1747.

Gonzalez-Reyes, A., H. Elliott, and D. St Johnston. 1995. Polarization of both major body axes in Drosophila by gurken- torpedo signaling. Nature 375: 654-658.

Han, J., Y. Jiang, Z. Li, V.V. Kravchenko, and R.J. Ulevitch. 1997. Activation of the transcription factor MEF2C by the MAP kinase p38 in inflammation. Nature 386: 296-299.

Han, S.J., K.Y. Choi, P.T. Brey, and W.J. Lee. 1998a. Molecular cloning and characterization of a Drosophila p38 mitogenactivated protein kinase. J. Biol. Chem. 273: 369-374.

Han, Z.S., H. Enslen, X. Hu, X. Meng, I.H. Wu, T. Barrett, R.J. Davis, and Y.T. Ip. 1998b. A conserved p38 mitogen-activated protein kinase pathway regulates Drosophila immunity gene expression. Mol. Cell. Biol. 18: 3527-3539.

Hawkins, N.C., C. Van Buskirk, U. Grossniklaus, and T. Schüpbach. 1997. Post-transcriptional regulation of gurken by encore is required for axis determination in Drosophila. Development 124: 4801-4810.

Herskowitz, I. 1995. MAP kinase pathways in yeast: For mating and more. Cell 80: 187-197.

Irie, K., Y. Gotoh, B.M. Yashar, B. Errede, E. Nishida, and K. Matsumoto. 1994. Stimulatory effects of yeast and mammalian 14-3-3 proteins on the Raf protein kinase. Science 265: $1716-1719$

Kawasaki, H., T. Morooka, S. Shimohama, J. Kimura, T. Hirano, Y. Gotoh, and E. Nishida. 1997.6 Activation and involvement of p38 mitogen-activated protein kinase in glutamateinduced apoptosis in rat cerebellar granule cells. J. Biol. Chem. 272: 18518-18521.

Kim-Ha, J., J.L. Smith, and P.M. MacDonald. 1991. oskar mRNA is localized to the posterior pole of the Drosophila oocyte. Cell 66: 23-34.

Kummer, J.L., P.K. Rao, and K.A. Heidenreich. 1997. Apoptosis induced by withdrawal of trophic factors is mediated by p38 mitogen-activated protein kinase. J. Biol. Chem. 272: 2049020494.

Kyriakis, J.M. and J. Avruch. 1996. Sounding the alarm: Protein kinase cascades activated by stress and inflammation. J. Biol. Chem. 271: 24313-24316.

Lasko, P.F. and M. Ashburner. 1990. Posterior localization of vasa protein correlates with, but is not sufficient for, pole cell development. Genes \& Dev. 4: 905-921.

Lehmann, R. 1995. Cell-cell signaling, microtubules, and the loss of symmetry in the Drosophila oocyte. Cell 83:353356.

Lin, A., A. Minden, H. Martinetto, F.X. Claret, C. Lange-Carter, F. Mercurio, G.L. Johnson, and M. Karin. 1995. Identification of a dual specificity kinase that activates jun kinases and p38-Mpk2. Science 268: 286-290.

Lindsley, D. and G. Zimm. 1992. The genome of Drosophila melanogaster. Academic Press, NY.

Maizels, E.T., J. Cottom, and P. Macdonald. 1996. aubergine enhances oskar translation in the Drosophila ovary. Development 122: 1631-1639.

Marin, R. and R.M. Tanguay. 1996. Stage-specific localization of the small heat shock protein Hsp27 during oogenesis in Drosophila melanogaster. Chromosoma 105: 142-149.

Musacchio, M. and N. Perrimon. 1996. The Drosophila kekkon genes: Novel members of both the leucine-rich repeat and immunoglobulin superfamilies expressed in the CNS. Dev. Biol. 178: 63-76.

Noselli, S. 1998. JNK signaling and morphogenesis in Drosophila. Trends Genet. 14: 33-38.

Perrimon, N. 1994. Signalling pathways initiated by receptor protein tyrosine kinases in Drosophila. Curr. Opin. Cell Biol. 6: 260-266.

Raingeaud, J., S. Gupta, J.S. Rogers, M. Dickens, J. Han, R.J. Ulevitch, and R.J. Davis. 1995. Pro-inflammatory cytokines and environmental stress cause p38 mitogen-activated pro- 
tein kinase activation by dual phosphorylation on tyrosine and threonine. J. Biol. Chem. 270: 7420-7426.

Raingeaud, J., A.J. Whitmarsh, T. Barrett, B. Derijard, and R.J. Davis. 1996. MKK3- and MKK6-regulated gene expression is mediated by the p38 mitogen-activated protein kinase signal transduction pathway. Mol. Cell. Biol. 16: 1247-1255.

Ray, R.P. and T. Schüpbach. 1996. Intercellular signaling and the polarization of body axes during Drosophila oogenesis. Genes \& Dev. 10: 1711-1723.

Rongo, C. and R. Lehmann. 1996. Regulated synthesis, transport and assembly of the Drosophila germ plasm. Trends Genet. 12: 102-109.

Roth, S., F.S. Neuman-Silberberg, G. Barcelo, and T. Schüpbach. 1995. cornichon and the Egf receptor signaling process are necessary for both anterior-posterior and dorsal-ventral pattern formation in Drosophila. Cell 81: 967-978.

Rouse, J., P. Cohen, S. Trigon, M. Morange, A. Alonso-Llamazares, D. Zamanillo, T. Hunt, and A.R. Nebreda. 1994. A novel kinase cascade triggered by stress and heat shock that stimulates MAPKAP kinase-2 and phosphorylation of the small heat shock proteins. Cell 78: 1027-1037.

Sapir, A., R. Schweitzer, and B.Z. Shilo. 1998. Sequential activation of the EGF receptor pathway during Drosophila oogenesis establishes the dorsoventral axis. Development 125: 191-200.

Spradling, A.C. 1993. Developmental genetics of oogenesis, In The development of Drosophila melanogaster (ed. C.M. Bate and A. Martinez-Arias). pp. 1-70. Cold Spring Harbor Laboratory Press, Cold Spring Harbor, NY.

St Johnston, D. 1993. Pole plasm and posterior group genes, In The development of Drosophila melanogaster (ed. C.M. Bate and A. Martinez-Arias). pp. 325-363. Cold Spring Harbor Laboratory Press, Cold Spring Harbor, NY.

Styhler, S., A. Nakamura, A. Swan, B. Suter, and P. Lasko. 1998. vasa is required for GURKEN accumulation in the oocyte, and is involved in oocyte differentiation and germline cyst development. Development 125: 1569-1578.

Symons, M. 1996. Rho family GTPases: The cytoskeleton and beyond. Trends Biochem. Sci. 21: 178-181.

Tomancak, P., A. Guichet, P. Zavorszky, and A. Ephrussi. 1998. Oocyte polarity depends on regulation of gurken by Vasa. Development 125: 1723-1732.

Vojtek, A.B. and J.A. Cooper. 1995. Rho family members: Activators of MAP kinase cascades. Cell 82: 527-529.

Wasserman, J.D. and M. Freeman. 1998. An autoregulatory cascade of EGF receptor signaling patterns the Drosophila egg. Cell 95: 355-364.

Wilson, J.E., J.E. Connell, and P. Macdonald. 1996. aubergine enhances oskar translation in the Drosophila ovary. Development 122: 1631-1639.

Xia, Z., M. Dickens, J. Raingeaud, R.J. Davis, and M.E. Greenberg. 1995. Opposing effects of ERK and JNK-p38 MAP kinases on apoptosis. Science 270: 1326-1331.

Yang, D., C. Tournier, M. Wysk, H.T. Lu, J. Xu, R.J. Davis, and R.A. Flavell. 1997. Targeted disruption of the MKK4 gene causes embryonic death, inhibition of c-Jun $\mathrm{NH}_{2}$-terminal kinase activation, and defects in AP-1 transcriptional activity. Proc. Nat1. Acad. Sci. 94: 13004-13009. 


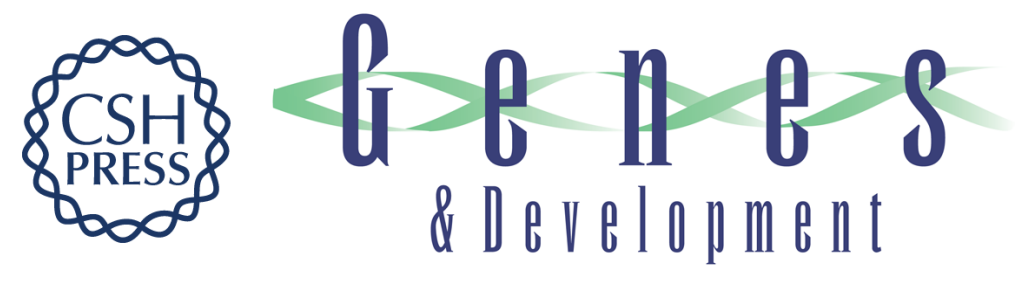

\section{The Drosophila p38 MAPK pathway is required during oogenesis for egg asymmetric development}

Magali Suzanne, Kenji Irie, Bruno Glise, et al.

Genes Dev. 1999, 13:

References This article cites 49 articles, 24 of which can be accessed free at:

http://genesdev.cshlp.org/content/13/11/1464.full.html\#ref-list-1

License

Email Alerting Receive free email alerts when new articles cite this article - sign up in the box at the top Service right corner of the article or click here.

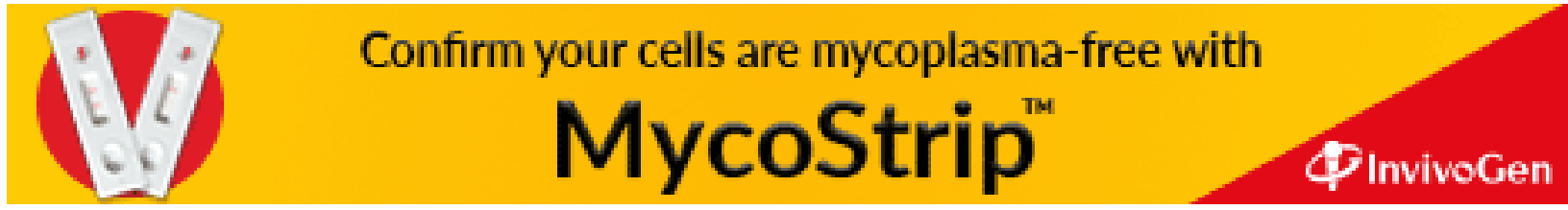

